\title{
Does exercise-induced myocardial ischaemia cause enhanced platelet activation and fibrin formation in patients with stable angina and severe coronary artery disease?
}

\author{
A. Gallino, A. Häberli, P. W. Straub, W. Steinbrunn, M. Turina and M. E. Rothlin \\ Department of Medicine and Cardiovascular Surgery, University Hospital of Zürich, Zürich and \\ Thrombosis Research Laboratory, Inselspital Bern, Switzerland
}

KEY WORDS: Beta thromboglobulin, fibrinopeptide A, stable angina, exercise-induced myocardial ischaemia.

In this study, betathromboglobulin (BTG) and fibrinopeptide $A(F P A)$ in peripheral venous blood were measured in 20 patients with stable angina pectoris before and immediately after exercise-induced myocardial ischaemia: in 5 of the 20 patients stable angina was associated with typical peripheral artery disease. A total of 10 patients with angiographically documented peripheral artery disease without angina and 10 normal volunteers were taken as control groups. BTG and FPA in the 15 patients with stable angina before exercise were $4 \mathrm{l} \pm 14 \mathrm{ng} \mathrm{ml}^{-1}$ and $2.3 \pm 0.9 \mathrm{ng} \mathrm{ml}^{-1}$ and were not statistically different from the values in normal controls; after exercise-induced myocardial ischaemia no significant increase occurred in these patients. Conversely, in the 5 patients with stable angina associated with peripheral artery disease BTG and FPA before exercise were $61 \pm 10 \mathrm{ng} \mathrm{ml}^{-1}$ and $3.5 \pm 0.8 \mathrm{ng} \mathrm{ml}^{-1}$ and increased to $114 \pm 14 \mathrm{ng} \mathrm{ml}^{-1}(\mathrm{P}<0.001)$ and $4 \cdot 1 \pm 0.5 \mathrm{ng} \mathrm{ml}^{-1}$ $(\mathrm{P}<0.01)$ : These results were similar to those found in the 10 patients with isolated peripheral artery disease.

We conclude that BTG and FPA in peripheral venous blood in patients with stable angina are not elevated either at rest or after exercise-induced myocardial ischaemia. Elevated values of $B T G$ and $F P A$ in patients with stable angina may reflect a major interaction between blood and atherosclerotic vessel wall, suggesting the presence of associated atherosclerotic lesions in peripheral artery disease.

\section{Introduction}

Coronary thrombosis is often associated with acute myocardial infarction ${ }^{[1-3]}$ and with unstable angina ${ }^{[4]}$. The measurement of betathromboglobulin (BTG) and fibrinopeptide A (FPA) in plasma are useful means of detecting enhanced platelet activation $^{[5]}$ and fibrin formation in vivo ${ }^{[6.7]}$. We recently reported that patients with unstable angina often present with elevated BTG and FPA levels ${ }^{[8,9]}$.

Discordant results have, however, been reported in regard to whether enhanced platelet activation and/or fibrin formation occur during exerciseinduced myocardial ischaemia in patients with stable angina ${ }^{[10-13]}$.

In the present study BTG and FPA were measured in 20 patients with stable angina before and immediately after exercise-induced myocardial

Submitted for publicauon on 31 July 1986 and in revised form 12 December 1986

Address for correspondence: Gallino Augusto, Department of Medicine, University Hospital, $\mathrm{CH}-8091$ Zürich, Switzerland. ischaemia. Particular attention was paid to the presence of concomitant peripheral artery disease.

\section{Methods}

PATIENTS

A total of 20 male patients with stable angina pectoris were admitted to the study. Criteria for admission were:

(1) presence of 3 vessel disease documented by coronary angiogram performed within 6 months before the study;

(2) pathological exercise ECG defined as horizontal or downsloping ST-depression of $\leqslant 2 \mathrm{~mm}$ in a precordial or inferior lead;

(3) absence of treatment with anticoagulants or platelet inhibitory agents within the preceding 3 months before the study;

(4) absence of acute myocardial infarction, thromboembolic events or other factors predisposing to coagulation disorders (neoplasia, severe infection, collagen disorders, glomerulonephritis).

Stable angina was defined as typical chest pain 
without an increase in frequency or severity, absence of chest pain at rest and absence of decrease in exercise tolerance during the 3 months preceding the study.

A total of 5 of the 20 patients complained of concomitant typical claudication of a lower limb associated with pathological ankle-arm pressure gradient measured by Doppler $(<0.5)$; in all the remaining 15 patients the ankle-arm pressure gradient was normal. Medication included betablockers in 16 patients, long-acting nitrates in 14 patients and calcium blockers in 12 patients. A total of 10 patients with severe peripheral artery disease documented angiographically without angina and 10 normal volunteers were taken as control groups.

\section{PLATELET AND COAGULATION TESTS}

Blood samples were taken by careful venepuncture through a 19 butterfly-needle immediately before and after exercise. All collections were done by the same investigator. For BTG measurements, $4.2 \mathrm{ml}$ of blood was collected into Thrombotect tubes (Abbott Laboratories, Chicago), placed immediately on ice and centrifuged within one hour at $2500 \mathrm{rpm}$ for 45 minutes at $4^{\circ} \mathrm{C}$; BTG was measured by radioimmunoassay ${ }^{[3]}$.

For the FPA measurements $4.5 \mathrm{ml}$ blood was collected into a plastic syringe containing $500 \mathrm{U}$ of heparin and $500 \mathrm{U}$ of aprotionin dissolved in $0.5 \mathrm{ml}$ of physiologic saline.

The samples were centrifuged within one hour for 30 minutes at $2500 \mathrm{rpm}$ at $4^{\circ} \mathrm{C}$. The plasma was stored at $-25^{\circ} \mathrm{C}$. FPA levels were measured by radioimmunoassay in plasma from which fibrinogen had been absorbed by bentonite ${ }^{[6,7]}$.

\section{EXERCISE PROTOCOL}

In the 20 patients with coronary artery disease and in the 10 controls, stress test exercise was performed on a bicycle ergometer, using a modified Bruce protocol. The ECG in $V_{1}, V_{5}$ and AVF leads was monitored using a computer assisted system (Marquette Case System). In patients with coronary artery disease the stress test was interrupted when the patient reported typical chest pain and there was a horizontal or descending ST-segment depression ( $60 \mathrm{~ms}$ after the $J$ point) of at least $2 \mathrm{~mm}$ in one of the monitored leads. The stress test in the 10 volunteers was interrupted at the moment of reaching maximal heart rate (215-age). In the 10 patients with isolated peripheral artery disease, the stress test exercise was performed using a treadmill: the stress test was interrupted when the patient

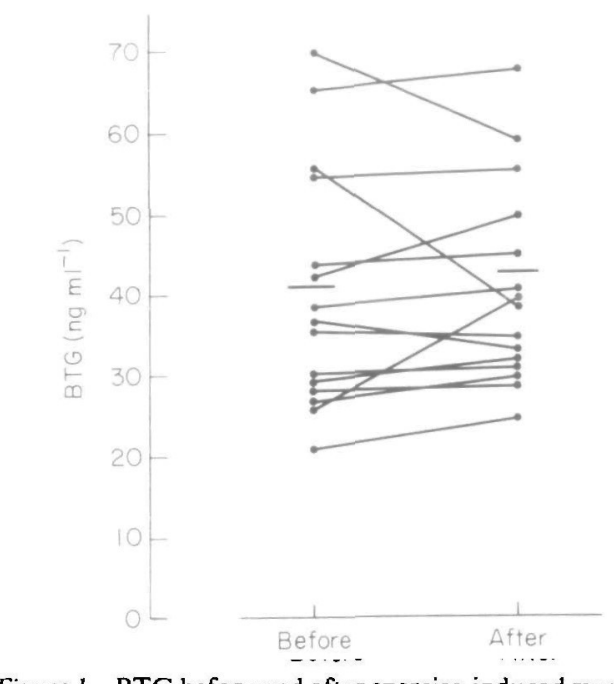

Figure I BTG before and after exercise-induced myocardial ischaemia in patients with stable angina without peripheral artery disease.

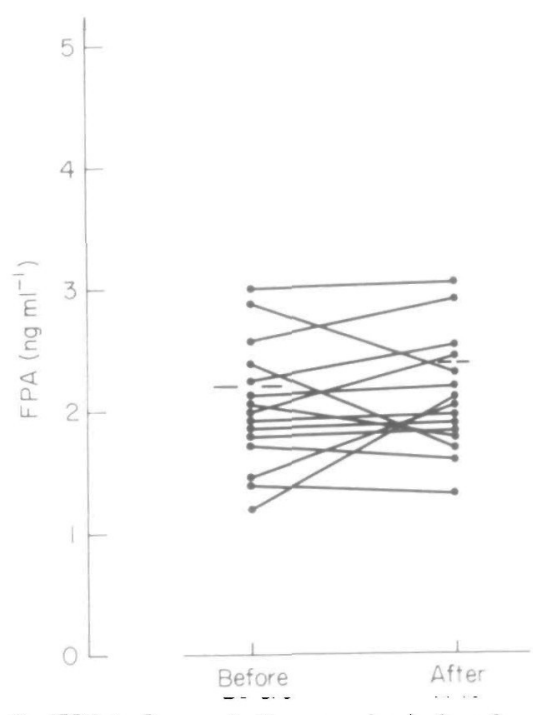

Figure 2 FPA before and after exercise-induced myocardial ischaemia in patients with stable angina without peripheral artery disease.

complained of severe typical claudication. None of these patients had chest pain and/or pathological ST-segment depression, Heart rate pressure product $\left(\mathrm{mmHg} \min ^{-1}\right)$ at peak exercise in these patients was $8 \pm 3.9 \times 10^{3}$ and significantly lower than in patients with $\operatorname{CAD}\left(16 \pm 4.1 \times 10^{3}\right)(P<0.01)$.

The paired $t$-test was used for comparison of the BTG and FPA values before and after exercise. The Student $t$-test was used for comparison of the heart rate-pressure products. 


\section{Results}

PATIENTS WITH CORONARY ARTERY DISEASE (CAD) AND WITHOUT PERIPHERAL ARTERY DISEASE (PAD)

In the 15 patients with CAD but without PAD, BTG and FPA levels in plasma at rest were $41 \pm 14 \mathrm{ng} \mathrm{ml}^{-1}$ and $2.3 \pm 0.9 \mathrm{ng} \mathrm{ml}^{-1}$, respectively and were slightly but not significantly elevated when compared to the values in normal controls (Table 2). Immediately after exercise-induced myocardial ischemia there was neither a significant increase of the BTG nor of the FPA level when compared to the values at rest (Figs 1 and 2).

\section{PATIENTS WITH CORONARY ARTERY DISEASE (CAD)} ASSOCIATED WITH PERIPHERAL ARTERY DISEASE (PAD)

The levels of BTG and FPA at rest in the 5 patients with CAD and PAD were $61 \pm 10 \mathrm{ng} \mathrm{ml}^{-1}$ and $3.5 \pm 0.8 \mathrm{ng} \mathrm{ml}^{-1}$, respectively, and signifcantly higher than in patients with CAD alone $(P<0.01)$ as well as in the controls $(P<0.01)$. After exercise-induced myocardial ischaemia BTG and FPA increased up to $114 \pm 14 \mathrm{ng} \mathrm{ml}^{-1}(P<0.0 \mathrm{l})$ and $4.1 \pm 0.5 \mathrm{ng} \mathrm{ml}^{-1}$, respectively $(P<0.01)$.

Table I Clinical and haemodynamic data of the patients with stable angina

\begin{tabular}{cccccc}
\hline$N$ & age (years) & NYHA & CAD & PAD & EF (\%) \\
20 & $56 \pm 10$ & 2.2 & 20 & 5 & $54 \pm 7$ \\
\hline
\end{tabular}

CAD (3 vd) = coronary artery disease with angiographically documented 3-vessel disease

PAD $=$ peripheral artery disease with typical claudication and pathological ankle-arm pressure gradient $(\leqslant 0.5)$

$\mathrm{EF}=$ ejection fraction
PATIENTS WITH PERIPHERAL ARTERY DISEASE (PAD)

The levels of BTG and FPA in plasma at rest in the 10 patients with PAD were $56 \pm 14 \mathrm{ng} \mathrm{ml}^{-1}$ and $3 \cdot 7 \pm 1 \cdot 2 \mathrm{ng} \mathrm{ml} \mathrm{m}^{-1}$. Immediately after calf pain limited exercise, levels of BTG and FPA increased up to $127 \pm 31 \mathrm{ng} \mathrm{ml}^{-1}(P<0.001)$ and $8 \cdot 0 \pm 4 \cdot 1 \mathrm{ng}$ $\mathrm{ml}^{-1}(P<0.01)$, respectively. BTG and FPA levels in the 10 volunteers were $28 \pm 13 \mathrm{ng} \mathrm{ml}-1$ and $1 \cdot 7 \pm 0.7 \mathrm{ng} \mathrm{ml}^{-1}$ and did not increase significantly after maximal effort exercise (Table 1).

\section{Discussion}

Our results indicate that enhanced platelet activation and fibrin formation do not occur in peripheral venous blood either at rest or immediately after exercise-induced myocardial ischaemia in patients with stable angina and severe coronary artery disease. These findings confirm the previous report of Marcella et al. ${ }^{[13]}$, but are in discrepancy with the results of several other authors ${ }^{[10-12]}$, who reported increased platelet activation in patients with angina.

Since we recently demonstrated that patients with severe peripheral artery disease often present evidence of increased fibrin formation in vivo ${ }^{[14]}$ we directed our attention to those patients with coronary artery disease and stable angina associated with clinical and haemodynamic signs of peripheral artery disease. In fact, of the small group of patients with angina associated with claudication all patients presented enhanced levels of BTG and FPA at rest and a further significant increase of these variables after exercise-induced myocardial ischaemia, reflecting the results of a control group of patients with angiographically documented severe peripheral artery disease (PAD). The absence

Table 2 BTG and FPA measurements (mean $\pm S D$ ) in patients with coronary artery disease (CAD) without and with peripheral artery disease ( $P A D$ ), in patients with $P A D$ alone and in normal volunteers before and immediately after exercise

\begin{tabular}{|c|c|c|c|c|c|}
\hline & \multirow{2}{*}{$N$} & \multicolumn{2}{|c|}{ BTG } & \multicolumn{2}{|c|}{ FPA } \\
\hline & & rest & exercise & rest & exercise \\
\hline $\begin{array}{c}\text { CAD } \\
\text { CAD+PAD } \\
\text { PAD } \\
\text { controls }\end{array}$ & $\begin{array}{r}15 \\
5 \\
10 \\
10\end{array}$ & $\begin{array}{l}41 \pm 14 \\
61 \pm 10 \dagger \\
56 \pm 14 \dagger \\
28 \pm 12\end{array}$ & $\begin{array}{c}42 \pm 15 \\
114 \pm 14^{*} \\
127 \pm 31^{*} \\
35 \pm 11\end{array}$ & $\begin{array}{l}2.3 \pm 0.9 \\
3.5 \pm 0.8 \dagger \\
3.7 \pm 1.2 \dagger \\
1.7 \pm 0.7\end{array}$ & $\begin{array}{l}2.4 \pm 0.9 \\
4 \cdot 1 \pm 0.5^{*} \\
8.0 \pm 4 \cdot 1^{*} \\
1.8 \pm 0.7\end{array}$ \\
\hline
\end{tabular}

* means $P<0.01$ when compared to the corresponding values at rest

tmeans $P<0.05$ when compared to the corresponding values in normal volunteers 
of angina and ST-segment depression in the patients with PAD does not exclude the presence of concomitant coronary artery disease (CAD). However, patients with severe CAD but without evidence of PAD did not present enhanced platelet activation and fibrin formation; whereas all patients with CAD and associated PAD had elevated BTG and FPA at rest and after exercise. Thus, the controversial reports on platelet activation and/ or fibrin formation in patients with angina may be explained by the presence or absence of patients with peripheral artery disease or - as we previously reported ${ }^{[8]}$ - by the inclusion of patients with unstable angina.

Platelet activation and fibrin formation may be enhanced in the coronary circulation without evidence in peripheral venous blood. Sampling of blood in coronary sinus, though often challenged because of artifactual activation due to the cardiac catheters, may give an answer to this question. The use of heparin bonded catheters may be a valid means solving this problem ${ }^{[15]}$.

\section{References}

[1] Davies MJ, Woolf, N, Robertson WB. Pathology of acute myocardial infarction with particular reference to occlusive coronary thrombi. Br Heart J 1976; 38: 659.

[2] De Wood MA, Spores J, Notske R. et al. Prevalence of total coronary occlusion during the early hours of transmural myocardial infarction. N Engl J Med 1980; 303: 897.

[3] Davies MJ, Thomas A. Thrombosis and acute coronary artery lesions in sudden cardiac ischemic death. $N$ Engl J Med 1984; 310: 1137
[4] Zack PM, Ischinger T, Aker UT el al. The occurrence of angiographically detected intracoronary thrombus in patients with unstable angina pectoris. Am Heart J 1984; 108: 1408

[5] Bolton AE, Ludlam CA, Moore S. et al. Three approaches to radioimmunoassay of betathromboglobulin. Br J Haematol 1976; 33: 323.

[6] Nossel HL. Radioimmunoassay of fibrinopeptides in relation to intravascular coagulation and thrombosis. $\mathrm{N}$ Engl J Med 1976; 295: 428.

[7] Hofmann V, Straub PW. A radioimmunoassay for the rapid measurement of human fibrinopeptide $A$. Thromb Res 1977; 11: 171

[8] Gallino A, Haeberli PH D, Baur HR, Straub PW. Fibrin formation and platelet aggregation in patients with severe coronary artery disease: relationship with the degree of myocardial ischemia. Circulation 1985; 72: 27.

[9] Sobel M, Salzman EW, Davies GC et al. Circulating platelet products in unstable angina pectoris. Circulation 1981; 63: 300 .

[10] Green LH, Seroppian E, Handin RI. Platelet activation during exercise-1nduced myocardial ischemia. $N$ Engl $J$ Med 302: 1980; 4, 193.

[11] Mehta J, Mehta P. Comparison of platelet function during exercise in normal subjects and coronary artery disease patients: Potential role of platelet activation in myocardial ischemia. Am Heart J 1982; 103: 49.

[12] Levine SP, Suarez AJ, Sorenson RR el al. Platelet factor 4 release during exercise in patients with coronary artery disease. Am J Haematol 1984; 17: 117

[13] Marcella JJ, Nichols AB, Johnson LL et al. Exerciseinduced myocardial ischemia in patients with coronary artery disease: Lack of evidence for platelet activation or fibrin formation in peripheral venous blood. J Am Coll Cardiol 1983; 1 (5): 1185.

[14] Gallino A, Haeberli A, Straub PW. Fibrinopeptide A excretion in urine in patients with atherosclerotic artery disease. Thromb Res 1985; 38: 237.

[15] Nichols AB, Owen J, Grossman BA et al. Effect of hepann bonding on catheter-induced fibrin formation and platelet activation. Circulation 1984; 70 (5): 843 . 